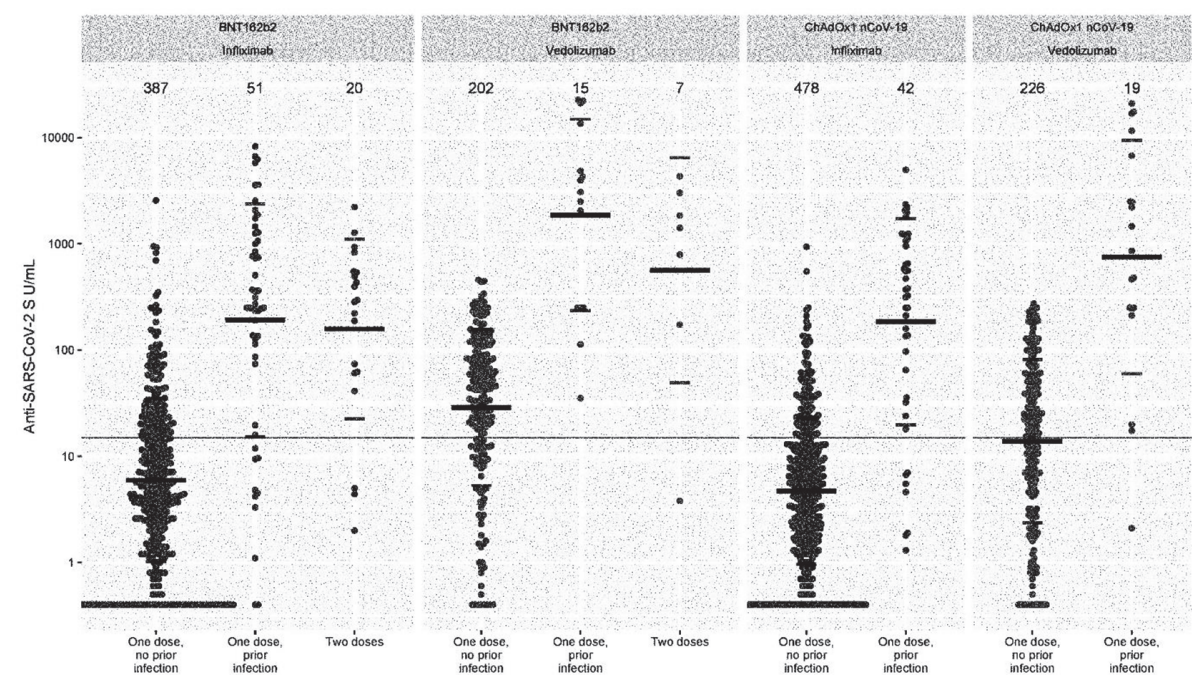

Abstract OFR-8 Figure 1

\section{OFR-9 AN RCT OF AUTOLOGOUS STEM-CELL TRANSPLANTATION IN TREATMENT REFRACTORY CROHN'S DISEASE (LOW-INTENSITY THERAPY EVALUATION): ASTICLITE}

\footnotetext{
${ }^{1}$ James Lindsay*, ${ }^{2}$ Shahida Din, ${ }^{3}$ Chris Hawkey, ${ }^{4}$ Danny Hind, ${ }^{5}$ Peter Irving, ${ }^{4}$ Alan Lobo, ${ }^{3}$ Yash Mahida, ${ }^{3}$ Gordon Moran, ${ }^{6}$ Miles Parkes, ${ }^{7}$ Jack Satsangi, ${ }^{8}$ Sree Subramanian, ${ }^{4}$ Lizzie Swaby, ${ }^{7}$ Simon Travis, ${ }^{4}$ John Snowden. ${ }^{1}$ Barts Health NHS Trust, London, UK; ${ }^{2}$ Western General Hospital, Edinburgh, UK; ${ }^{3}$ University of Nottingham, Nottingham, UK; ${ }^{4}$ University of Sheffield, Sheffield, UK; ${ }^{5}$ Guy's and St Thomas' Hospital, London, UK; ${ }^{6}$ Cambridge University Hospitals, Cambridge, UK; ${ }^{7}$ Oxford University Hospitals, Oxford, UK; ${ }^{8}$ Royal Liverpool University Hospital, Liverpool, UK
}

\subsection{6/gutjnl-2021-BSG.6}

Introduction Reports of benefit from HSCT were tempered by the ASTIC trial which failed its ambitious primary endpoint and reported high toxicity. Subsequent reports suggest that HSCT achieves complete mucosal healing in 50\%, and that toxicity relates to the cyclophosphamide dose.

Design A UK multi-site RCT comparing low intensity HSCT with standard of care (SOC) in patients with active CD at endoscopy (SESCD ulcer score $\geq 2$ ) refractory to 2 biologic classes. Planned recruitment was 99 patients randomised 2:1 to HSCT versus SOC. The primary endpoint was centrally read endoscopic remission (SESCD ulcer subscore of 0) without requirement for surgery or death at week 48 .

Results The trial was halted due to unexpected SAE after 23 patients (13 HSCT, 10 SOC) had been randomised. The coronavirus pandemic prevented some outcome assessments. Patients had advanced disease: mean (SD) CD duration 13.9 (7) years; CDAI at baseline 337.5 (182.4) with 20 (91\%) having undergone surgery and $9(43 \%)$ having a stoma. All patients contributed to the safety analysis. The primary outcome using central reading was available for 7/10 HSCT and 6/9 SOC patients. Absence of endoscopic ulceration without surgery or death was reported in $3 / 7$ (43\%) HSCT patients compared to $0 / 9(0 \%)$ SOC patients. Centrally read SESCD (mean (SD)) was $11.8(8.7)$ and 10.1 (5.7) at baseline compared to $2.8(2.9)$ and $18.7(9.1)$ at week 48 in the HSCT and SOC groups respectively. Clinical remission (CDAI <150) occurred in $57 \%$ and $0 \%$ of patients in the HSCT and SOC groups at week 48. SAE were more frequent after HSCT (39 in $13(100 \%)$ patients) than SOC (15 in 4 (40\%) patients).
Importantly, 10 SUSARs were reported in 6 HSCT patients including 3 cases of delayed renal failure due to thrombotic microangiopathy (TMA). Two patients in the HSCT arm died. Conclusion HSCT using a low intensity regimen results in meaningful reduction in endoscopic disease activity with some patients experiencing resolution of ulceration. However, the incidence of serious adverse events makes the regimen used in this trial unsuitable for future clinical use.

This project $(15 / 178 / 09)$ is funded by the Efficacy and Mechanism Evaluation (EME) Programme, an MRC and NIHR partnership. The views expressed in this publication are those of the author(s) and not necessarily those of the MRC, NIHR or the Department of Health and Social Care.

\section{OFR-10 ADMISSION MODEL FOR INTENSIFICATION OF THERAPY IN ACUTE SEVERE COLITIS (ADMIT-ASC)}

${ }^{1}$ Alex Adams*, ${ }^{1}$ Vipin Gupta, ${ }^{2}$ Waled Mohsen, ${ }^{1}$ Thomas Chapman, ${ }^{2}$ Deloshaan Subhaharan, ${ }^{2}$ Pradeep Ramaswamy, ${ }^{1}$ Oxford IBD Cohort Investigators, ${ }^{3}$ Vineet Ahuja, ${ }^{1}$ Simon Travis, ${ }^{1}$ Jack Satsangi. ${ }^{1}$ Translational Gastroenterology Unit, University of Oxford, Oxford, UK; ${ }^{2}$ Digestive Diseases Unit, Gold Coast University Hospital, Australia; ${ }^{3}$ Department of Gastroenterology, All India Institute of Medical Sciences, New Delhi, India

\subsection{6/gutjinl-2021-BSG.7}

Introduction Acute severe colitis (ASC) is an important cause of morbidity and mortality in UC, requiring hospitalisation and often colectomy. Accepted management is protocolised response assessment at Day 3 of IV steroid treatment. If steroid non-responders could be identified at all earlier stage, intensification may be possible prior to Day 3. It is also unclear whether ASC outcomes have chaned over the past 25 years and we aimed to examine this.

Methods We examined ASC cohorts across 3 continents to produce an accurate predictor of steroid response. All patients received protocolised treatment including first-line IV corticosteroids, and endoscopic (UCEIS) scoring. Factors associated with requirement for rescue therapy, colectomy during admission, and the following year, and a composite measure of steroid response were identified by logistic regression in 131 adult ASC admissions in Oxford, UK, between 2015-9. 
Predictive models comprising combinations of clinical parameters, blood results, and endoscopy findings were ranked by AIC with a final model selected with an emphasis on simplicity and ease of implementation. This model was validated in 110 patients admitted to GCUH, Australia between 2015-20 and 62 patients admitted to AIIMS, India between 2015-8. The Oxford cohort were compared with data from the same unit in 1992-3 (Travis et al, Gut 1996; 38: 905-10).

Results Admission CRP, albumin, and UCEIS scores predicted steroid non-response at day 3 (FDR $p=0.00066,0.0066$, and 0.015). A 4-point model was developed involving CRP $\geq 100 \mathrm{mg} / \mathrm{L}$, albumin $\leq 25 \mathrm{~g} / \mathrm{L}$, UCEIS $\geq 4$, or $\geq 7$. Scoring 0 or 4 was $100 \%$ accurate in all cohorts. $19.5 \%$ of patients scored 3 or 4 of whom $83 \%$ in Oxford, $85 \%$ in GCUH and $80 \%$ in AIIMS failed steroid treatment. In the combined validation cohorts the PPV for steroid failure was 0.84 (0.70-0.98), OR 11.9 (10.8-13), with the number needed to screen 8.2 (5.813.7).

In the current Oxford cohort $54 \%$ of patients received rescue therapy (27\% ciclosporin, $27 \%$ anti-TNF), increased from $27 \%$ ciclosporin in 1992-3, $\mathrm{p}=0.0015$. Colectomy during admission halved ( $15 \%$ vs $29 \%, \mathrm{p}=0.033)$, but an eight-fold increased risk of colectomy in the following year is still seen after a partial steroid response.

Conclusions Our model identifies on admission a subset of patients very unlikely to respond to steroid treatment and allows stratification for early intensification of management. In 25 years colectomy rates for ASC have halved while secondline therapy use has doubled. Long-term outcomes remain poorer in patients who do not clearly respond to steroids.

\section{OMO-1 EPIGENETIC ALTERATIONS IN IBD: DEFINING GEOGRAPHICAL, GENETIC, AND IMMUNE- INFLAMMATORY INFLUENCES ON THE CIRCULATING METHYLOME}

${ }^{1}$ Rahul Kalla*, ${ }^{2}$ Alex Adams, ${ }^{3}$ Jan Nowak, ${ }^{4}$ Daniel Bergemalm, ${ }^{5}$ Simen Vatn, ${ }^{1}$ Nicholas Ventham, ${ }^{6}$ Nicholas Kennedy, ${ }^{5}$ Petr Ricanek, ${ }^{5}$ Jonas Lindstrom, ${ }^{7}$ Marieke Pierik, ${ }^{8,13}$ Mauro D'Amato, ${ }^{9}$ Fernando Gomollon, ${ }^{5}$ Christine Olbjorn, ${ }^{10}$ Rebecca Richmond, ${ }^{10}$ Caroline Relton, ${ }^{11}$ Johan Soderholm, ${ }^{5,12}$ Jorgen Jahnsen, ${ }^{5}$ Morten Vatn, ${ }^{4}$ Jonas Halfvarson, ${ }^{2}$ Jack Satsangi. ' University Of Edinburgh, Edinburgh, UK; ${ }^{2}$ University of Oxford, Oxford, UK; ${ }^{3}$ Poznan University of Medical Sciences, Poznan, Poland; ${ }^{4}$ Orebro University, Orebro, Sweden; ${ }^{5}$ University of Oslo, Oslo, Norway; ${ }^{6}$ University of Exeter, Exeter, UK; ${ }^{7}$ Maastricht University Medical Centre, Maastricht, Netherlands; ${ }^{8}$ BioCruces Health Research Institute and IKERBASQUE, Bilbao, Spain; 'HCU 'Lozano Blesa,' IIS Aragón, Zaragosa, Spain; ${ }^{10}$ University of Bristol, Bristol, UK; ${ }^{11}$ University of Linkoping, Linkoping, Sweden; ${ }^{12}$ Akershus University Hospital, Akershus, Norway; ${ }^{13}$ Monash University, Melbourne, Australia

\subsection{6/gutjnl-2021-BSG.8}

Introduction DNA methylation may provide critical insights into gene-environment interactions in inflammatory bowel disease (IBD).

Methods Using the multi-centre IBD Character inception cohort (295 controls, 154 CD, 161 UC, 28 IBD-U), epigenome-wide methylation was profiled using Illumina HumanMethylation450 platform. Differentially methylated position analysis was performed using age, sex and cell proportions as covariates. Integration of paired genomic and transcriptomic layers was done with Multi-Omics Factor Analysis v2 (MOFA). Unsupervised principal component analyses were performed to examine correlates of treatment escalation and clinical predictors of disease severity.
Results We report 137 differentially methylated positions (DMP) in whole blood in IBD, including VMP1/MIR21 $\left(\mathrm{p}=9.11 \times 10^{-15}\right)$ and RPS6KA2 $\left(6.43 \times 10^{-13}\right)$; with consistency seen across Scandinavia and UK. Cell of origin analysis preferentially implicated the monocyte lineage. Dysregulated loci demonstrate strong genetic influence, notably VMP1 $\left(\mathrm{p}=1.53 \times 10^{-15}\right)$. Age acceleration is seen in IBD (coefficient $\left.0.94, \mathrm{p}<2.2 \times 10^{-16}\right)$. Several immuno-active genes demonstrated highly significant correlations between methylation and gene expression in IBD, in particular OSM: IBD $r-0.32$, $p$ $3.64 \times 10^{-7}$ vs. non-IBD $\mathrm{r}-0.14, \mathrm{p}=0.77$ ). Multi-omic integration of methylome, genome and transcriptome also identified specific pathways that associate with immune activation, response and regulation at disease inception. At follow up, a signature of 3 DMPs (TAP1, TESPA1, RPTOR) associated with treatment escalation to biological agents or surgery (hazard ratio of 5.19 (CI:2.14-12.56, logrank $\left.\mathrm{p}=9.70 \times 10^{-4}\right)$.

Conclusion This study highlights the stability of the IBD-specific circulating methylome across regions with shared ancestry. Through integrative multi-omic analyses we identify key proinflammatory genes that are upregulated in IBD at inception. Furthermore, differential methylation within certain genes such as TAP1 associate with disease course over time.

\section{OMO-2 FILGOTINIB EFFICACY IN PATIENTS WITH ULCERATIVE COLITIS BY LINE OF THERAPY: PHASE 2B/3 SELECTION RESULTS}

${ }^{1}$ Ben Morison*, ${ }^{2}$ Lauren Peyrin-Biroulet, ${ }^{3,4} 4$ ris Dotan, ${ }^{5}$ Toshifumi Hibi, ${ }^{6}$ Virginia Taliadouros, ${ }^{6}$ Alessandra Oortwijn, ${ }^{7}$ Sally Zhao, ${ }^{7}$ Jie Zhang, ${ }^{7}$ Jeremy Hsieh, ${ }^{8}$ Brian Feagan. ${ }^{1}$ Norfolk and Norwich University Hospital, Norfolk, UK; ${ }^{2}$ Inflammatory Bowel Disease Unit, Nancy University Hospital, France; ${ }^{3}$ Division of Gastroenterology, Rabin Medical Center, Israel; ${ }^{4}$ Sackler Faculty of Medicine, Tel Aviv University, Israel; ${ }^{5}$ Kitasato Institute Hospital, Kitasato University, Japan; ${ }^{6}$ Galapagos NV, Leiden, Netherlands; ${ }^{7}$ Gilead Sciences, Inc., Foster City, USA; ${ }^{8}$ Western University, London, Canada

\subsection{6/gutjnl-2021-BSG.9}

Introduction Filgotinib (FIL) is a once-daily, oral, Janus kinase 1 preferential inhibitor. We assessed the efficacy of FIL in biologic (bio)-naïve and bio-experienced patients with UC, and in bioexperienced patients with failure of 1 or $\geq 2$ biologics or 1 or 2 mechanisms of action (MoAs).

Methods SELECTION (NCT02914522) was a phase 2b/3 double-blind, randomised, placebo-controlled trial comprising two induction studies and a maintenance study. Adults (18-75 years) with moderately to severely active UC were randomised 2:2:1 to FIL $200 \mathrm{mg}$, FIL $100 \mathrm{mg}$ or placebo (PBO) once daily for 11 weeks in Induction Study A (bio-naïve) and B (bio-experienced). Patients in either clinical remission or Mayo Clinic Score (MCS) response at week 10 (responders) could enter the Maintenance Study. Responders who received induction FIL were re-randomised $2: 1$ to continue their induction regimen or $\mathrm{PBO}$ through week 58. Responders who received induction $\mathrm{PBO}$ continued $\mathrm{PBO}$. We assessed clinical remission and MCS response at weeks 10 and 58 in bio-naïve patients and bio-experienced patients with failure of 1 or $\geq 2$ biologics and 1 or 2 MoAs (TNF antagonists and vedolizumab). All $p$ values for subgroup analyses are nominal.

Results Week 10 clinical remission was achieved by a significantly higher proportion of bio-naïve and -experienced patients treated with FIL $200 \mathrm{mg}$ than PBO. A higher proportion of bio-experienced patients with 1 biologic or MoA failure treated with FIL $200 \mathrm{mg}$ than PBO achieved clinical 